\title{
De l'approche conventionnelle à l'approche communicationnelle des mondes organisationnels
}

Jean-Luc Bouillon

\section{(2) OpenEdition}

1 Journals

Édition électronique

URL : http://journals.openedition.org/communicationorganisation/2912

DOI : 10.4000/communicationorganisation.2912

ISSN : $1775-3546$

Éditeur

Presses universitaires de Bordeaux

Édition imprimée

Date de publication : 1 mai 2004

ISSN : 1168-5549

Référence électronique

Jean-Luc Bouillon, « De l'approche conventionnelle à l'approche communicationnelle des mondes organisationnels », Communication et organisation [En ligne], 24 | 2004, mis en ligne le 27 mars 2012, consulté le 19 avril 2019. URL : http://journals.openedition.org/communicationorganisation/2912 ; DOI : 10.4000/communicationorganisation.2912

Ce document a été généré automatiquement le 19 avril 2019

(c) Presses universitaires de Bordeaux 


\title{
De l'approche conventionnelle à l'approche communicationnelle des mondes organisationnels
}

\author{
Jean-Luc Bouillon
}

1 La notion de monde organisationnel fait en premier lieu référence aux réalités vécues par des acteurs, dans leur travail quotidien, dans les équipes ou les groupes projets auxquels ils participent. Parallèlement, cette notion renvoie aux formules permettant de décrire et de reconstruire de manière cohérente les réalités professionnelles à partir d'une perspective particulière. Un monde organisationnel désigne ce qui est conceptualisé comme tel. cette référence servant de repère à ceux qui le vivent quotidiennement. C'est ainsi à de multiples conceptions parallèles de l'organisation que l'on se trouve confronté : le monde organisationnel des services commerciaux n'est pas celui des opérateurs de production, ni celui des ingénieurs de recherche et développement ou des actionnaires. Dans l'organisation l'ordiste de la production, ces mondes correspondaient à des fonctions organisationnel les et à des règles de fonctionnement bien spécifiques, qui tendent à être remises en question. Normalisation liée à l'assurance qualité, développement des Technologies d'Information et de Communication (TIC) semblent ainsi conduire à une harmonisation, voire à une fusion des mondes organisationnels au nom de l'efficacité. Comment saisir une telle tendance? Dans un premier temps, nous nous efforcerons de définir la notion de monde organisationnel en nous appuyant sur le cadre théorique développé par les socio-économistes des conventions, tout particulièrement Luc Boltanski. Laurent Thévenot et plus récemment Eve Chiapello1. Nous chercherons également à la resituer dans une perspective communicationnelle. Nous examinerons ensuite comment la phase actuelle de l'évolution économique, marquée par un accroissement de la rationalisation des organisations et du travail touchant tout particulièrement les dimensions cognitives. se caractérise par une volonté d'intégrer les mondes organisationnels et délimiter les confrontations qui les caractérisent. Ce mouvement prend forme au travers de la généralisation des démarches projets et s'appuie sur une conception réticulaire de l'organisation. Enfin, nous 
analyserons comment les portails d'entreprise dédiés à la gestion des connaissances semblent matérialiser celle vision et constituer l'un des principaux vecteurs de l'harmonisation des mondes organisationnels.

\section{Les mondes organisationnels en débat}

\section{Logiques d'action et de coordination dans les organisations}

2 L'une des conceptualisations les plus aboulies des mondes organisationnels nous semble portée par les travaux conduits depuis plus de quinze ans au sein du courant français de la socio-économie des conventions, dans le prolongement des Economies de la grandeur. Cette approche fait naturellement l'objet de contestations parfois virulentes ${ }^{2}$ mais n'en présente pas moins un caractère particulièrement stimulant au travers de l'ambition même dont elle fait preuve, en proposant un cadre théorique ayant vocation à problématiser des transformations sociétales d'ensemble, intégrant le singulier et le général dans une perspective historique.

3 Contrairement à la vision des approches économiques standard, les organisations ne peuvent se résumer à des structures juridiques assurant la circulation et le traitement des informations: elles ne sont pas établies une fois pour toutes, mais construites quotidiennement, au gré de leur existence économique, sociale, ainsi que des évolutions de l'environnement. La notion de convention désigne ainsi un processus de mise en cohérence d'actions individuelles, dans lequel se mêlent application ou adaptation de règles, conflits, négociations, échanges interpersonnels, ainsi que le résultat de ce processus en ternies de mise en place d'une organisation cohérente. C'est ainsi un " dispositif cognitif collectif» qui s'élabore en situation, et permet la coordination et le fonctionnement organisationnel quotidien. Les actions et les échanges dépendent directement de la caractérisation du contexte dans lequel ils ont lieu, ce qui implique discussions, confrontations, arbitrages, permettant de déboucher, à défaut d'accord stable, sur des compromis, ou une non incompatibilité temporaire de logiques d'action ${ }^{3}$.

Les travaux initiés par Luc Boltanski et Laurent Thévenot permettent précisément de mieux identifier les logiques d'actions et les processus d'émergence des dispositifs cognitifs collectifs. A partir d'une analyse approfondie d'œuvres fondatrices de la philosophie politique occidentale, ces auteurs identifient six cités cohérentes, associées à une motivation idéale-typique de l'action individuelle et définies par un principe supérieur communément reconnu comme légitime. Caractérisé par des valeurs, des représentations et donnant lieu à des concepts et à un vocabulaire spécifique, chaque principe renvoie à une vision particulière du monde, qui permet d'interpréter, d'expliquer, de contester, de justifier actions, situations et comportements en référence aux valeurs spécifiques d'une cité, par delà leur singularité.

De la rencontre entre ces cités et une sélection d'œuvres représentatives de la littérature managériale, rendant compte des représentations et des cadres idéologiques en vigueur chez les dirigeants d'entreprise, naissent des mondes. Ces derniers caractérisent les multiples modalités de coordination et d'action par lesquelles se construisent et évoluent les organisations. Le monde de l'inspiration est ainsi fondé sur le principe de la créativité liée à l'inspiration individuelle. La passion, l'imagination, l'authenticité attachées à la personnalité du créateur permettent de justifier les actions. Les ligures de référence sont - entre autres - l'artiste, l'artisan, le publicitaire, le chercheur. Pour sa part, le monde 
domestique repose sur le respect de la tradition et de l'ordre établi tels qu'on les trouve dans une structure hiérarchique ou entre les membres d'un clan familial. L'appartenance des individus à une communauté, leur position dans une chaîne de dépendances personnelles, leur fidélité constituent les principales valeurs. L'organisation paternaliste, les corporations professionnelles sont par exemple fortement marquées par cette logique. Dans le monde de l'opinion, c'est la reconnaissance publique qui est essentielle. Les valeurs essentielles sont l'image, le crédit inspiré à autrui, le prestige. Le monde civique repose pour su part sur la primauté de l'intérêt général. La capacité à se mobiliser pour la collectivité au-delà de ses propres intérêts, le dévouement à l'organisation, à l'équipe, qui transcende les individus, sert alors à justifier et à évaluer individus et actions. A l'inverse, le monde marchand repose sur l'enrichissement individuel par la vente de marchandises auprès de clients sur un marché. L'accroissement du chiffre d'affaires et du profit justifie et permet déjuger de la pertinence d'une action ou d'une personne. Enfin, dans le inonde industriel, l'efficacité technique et organisationnel le constitue le critère déterminent. Les valeurs centrales sont l'amélioration de la productivité, de lu Habilité des processus. Le bureau des méthodes taylorien, l'organisation en flux tendus dite post-taylorienne. sont autant d'éléments représentatifs de ce monde.

6 En s'appuyant sur le cadre théorique des économies de la grandeur, il est ainsi possible d'identifier six mondes organisationnels idéaux-typiques, au sein desquels les actions et leur coordination peuvent être motivées et justifiées par le désir de créer, le respect de l'ordre établi, l'amélioration de l'image, l'intérêt collectif, le profit et l'efficacité technique. Ces mondes sont pour partie incompatibles, mais de leur mise en cohérence et de leur stabilisation temporaire dépend l'existence et la pérennité d'une organisation.

\section{Communication et mondes organisationnels}

7 Si l'approche conventionnelle permet d'isoler des idéaux-types des mondes organisationnels et de mettre en évidence les difficultés de leur mise en cohérence, elle demeure très discrète, du moins dans sa version de 1991, sur les implications du système économique où évoluent les organisations, et sur les rapports sociaux qui le caractérisent. Or, articulations entre mondes d'action ne se déploient pas dans un univers abstrait et éthéré, mais dans un système capitaliste, qui, faut-il le rappeler, repose sur la capacité des organisations privées à dégager un profit à partir de l'engagement du capital dont elles disposent dans le circuit économique. La génération de profit implique d'être compétitif avec les autres acteurs dans un marché concurrentiel. Par ailleurs, l'organisation capitaliste repose également sur un rapport social de type salarial, par lequel ceux nombreux - qui ne disposent pas du capital tirent leurs revenus de la vente de leur travail et se trouve soumis à l'autorité des détenteurs du capital. Un acteur au sein d'une organisation n'est pas un simple individu: il est soumis à des pressions économiques et sociales qui les contraignent à accepter les principes de l'organisation.

Les responsables des organisations équipes dirigeantes, consultants, actionnaires vont chercher par tous les moyens à mettre en cohérence les mondes organisationnels pour assurer le fonctionnement organisationnel. Pour cela, la pression économique pesant sur les salariés joue un rôle essentiel, mais n'est pas toujours suffisante. Dès lors que les travaux ne sont pas totalement prescrits, la présence d'un système d'interprétation du monde est nécessaire pour fournir les arguments et les valeurs justifiant une forte implication et une adhésion active. Le système économique doit ainsi construire un sens à 
son propre fonctionnement ${ }^{4}$. Les postulats généraux doivent être déclinés au sein des organisations pour assurer la mise en cohésion des mondes au quotidien : tel est le but des dispositifs institutionnels, techniques et idéologiques qui orientent les activités et leur coordination. Une éventuelle intégration des mondes organisationnels s'inscrit dans ce cadre : comment toutefois, la conceptualiser, et l'utiliser pour conduire des travaux empiriques?

Dans la grille des économies de la grandeur, les phénomènes de communication qui se déroulent au sein des organisations apparaissent en permanence en filigrane il est question de négociation, de disputes, de compromis, de justifications mais ne sont jamais appréhendés en tant que tels. Au-delà des cloisonnements disciplinaires, l'une îles principales explications de cette situation tient certainement au fait que les multiples travaux réalisés depuis plus de dix ans dans le champ des communications organisationnelles ${ }^{5}$ manquent de visibilité et ne parviennent pas à imposer des systèmes cohérents d'interprétation des organisations à partir des phénomènes de communication qui les traversent, line perspective communicationnelle nous semblerait pourtant constituer une piste pertinente pour penser la mise en relations des logiques d'actions idéales-typiques correspondant aux mondes organisationnels avec les enjeux et les contraintes économiques globales qui caractérisent les organisations. De fait, il existe de multiples manières de concevoir une organisation dans une perspective communicationnelle. Nous retiendrons l'une d'entre elles 6 , considérant qu'il est possible d'appréhender une organisation en mettant en relation trois niveaux interdépendants: d'une part les situations de communication qui se déroulent localement, ensuite les processus de communication, associés aux processus industriels et administratifs et enfin les politiques de communication, qui visent à produire une description cohérente de l'organisation ainsi que les cadres d'interprétation susceptibles de contribuer à l'orientation des actions.

\section{Vers l'intégration des mondes organisationnels?}

\section{Les rationalisations cognitives}

10 Historiquement, l'accroissement de la profitabilité s'est appuyé sur un processus continu de rationalisation du travail et des organisations, visant à accroître leur efficacité. La période actuelle, parfois qualifiée d'économie cognitive ${ }^{7}$ se caractérise par une extension de la rationalisation de la réalisation des tâches, caractéristique de la période taylorienne, aux activités cognitives ${ }^{8}$.Ces dernières tendent à acquérir une existence autonome dans la pensée économique comme dans la réalité des systèmes productifs, leur rôle dans la production de richesses apparaissant désormais primordial.

Les rationalisations cognitives portent en premier lieu sur les données indispensables au fonctionnement de toute organisation marchande et. de plus en plus souvent, non marchande. L'objectif est de favoriser la circulation et le traitement d'informations liées à la gestion administrative et comptable, aux transactions (commandes, paiements, logistique, prestations de services...) et à la connaissance des clients et du marché, dans un contexte où l'individualisation des comportements de consommation se traduit par une personnalisation accrue des offres commerciales. Cette rationalisation comporte un versant organisationnel remontant au début des années 1980, associé au développement des nouvelles organisations en flux tendus, plaçant le client au centre du système économique et cherchant à limiter au maximum les coûts de fonctionnement, et de 
stockage. La qualité de la circulation et du traitement des informations à chaque niveau est essentielle au bon fonctionnement du système: le système s'appuie à présent largement sur les TIC. et notamment sur les Progiciels de Gestion Intégrés (ERP) et leurs différents modules consacrés, par exemple à la gestion de la relation avec les clients ou avec les fournisseurs (e-CRM et SRM).

En second lieu, les rationalisations cognitives portent sur les méthodes de travail, les modes opératoires, et les démarches professionnelles permettant la production effective de biens ou de services ainsi que leur commercialisation. Au-delà de l'amélioration de l'efficacité économique par une meilleure maîtrise des processus, l'objectif visé est la conservation des savoirs, des savoir-faire et de l'expérience de l'organisation. Il s'agit de codifier les savoirs (D. Foray. B.A. Lundvall. 1998). c'est-à-dire les dissocier des personnes et des groupes qui les détiennent afin de favoriser leur transmission et leur acquisition par les autres acteurs qui seraient amenés à les utiliser. Outre le renforcement du niveau de prescription du travail à l'usage des salariés, l'enjeu est de présenter un processus de production lisible et transparent à de futurs donneurs d'ordre et de favoriser le retour d'expérience par une meilleure maîtrise des processus productifs. Les démarches de certification aux normes d'assurance qualité ISO 9000 constituent l'une des principales ligures de ces rationalisations cognitives.

Cependant, savoirs et savoir-faire ne peuvent en effet être considérés comme des ressources comme les autres: ils ne peuvent être complètement codifiés, et conservent une dimension irréductiblement tacite. Même dans le cas où ils sont très largement transcrits, les conditions (techniques, sociales, organisationnelles...) de leur mise en œuvre varient en permanence, à chaque Ibis qu'ils sont mobilisés, surtout dans les nouvelles organisations productives marquées par l'incertitude. Ceci implique des ajustements, des adaptations, débouchant d'ailleurs sur la production de nouvelles connaissances en situation. La valeur du capital cognitif cette notion est d'ailleurs contestable n'est pas seulement liée à la capacité à conserver des savoirs, mais dépend aussi de l'aptitude des acteurs d'une organisation à les mobiliser collectivement, c'est-àdire à les adapter aux situations rencontrées dans le cadre du travail ${ }^{9}$. C'est ainsi que la rationalisation cognitive porte plus largement sur les processus de mobilisation des savoirs, c'est-à-dire sur les capacités d'individus et de collectifs de travail à produire, retrouver, à les élaborer, à les adapter, à les mettre en œuvre dans le cadre d'une situation de travail collective qui peut rapprocher des personnes appartenant à une même organisation, ou même à plusieurs organisations.

On l'aura compris, ce sont les situations de communication qui doivent être gérées pour favoriser leur intégration dans les processus de l'organisation, au travers des différents mondes organisationnels. La capacité à travailler collectivement, à communiquer, à mobiliser des connaissances et à s'auto-organiser devient ainsi une condition de l'efficacité économique et une source essentielle de productivité, que l'on considère des activités de production en flux tendus où il faut rapidement résoudre des dysfonctionnements ou dans des activités de conception. Ces compétences permettent de respecter strictement les normes existantes et de les adapter ou de les compléter dans le bon sens lorsque c'est nécessaire, et assure également une bonne exploitation des multiples informations fournies sur les activités de l'organisation. 


\section{Projets, réseaux et intégration des mondes organisationnels}

15 L'actuelle phase de l'évolution économique implique donc un fonctionnement intégré des différents mondes, quels que soient leurs incompatibilités potentielles, l'économie cognitive et l'extension des rationalisations qui la caractérisent s'inscrivent en effet dans le cadre des transformations subies par le régime d'accumulation pour s'adapter aux nouvelles contraintes de valorisation du capital. L'accentuation de la concurrence liée à la mondialisation s'est traduite par une externalisation des activités qui n'entrent pas dans le métier de base des organisations, par un renforcement de la flexibilité des processus productifs, et par un développement de partenariats ponctuels : l'interconnexion entre fournisseurs et sous traitants, dans le cadre d'accords plus ou moins pérennes tend à dissiper les frontières entre l'organisation et son environnement ${ }^{10}$. Dans ce contexte, le client devient la ligure centrale y compris au sein des organisations avec le développement de la sous-traitance interne, au point qu'il tend à prendre la place d'une hiérarchie allégée pour davantage de souplesse et également pour des raisons d'économie salariale. Cette évolution implique une organisation moins rigide et hiérarchique, susceptible de se mobiliser de manière réactive pour faire face aux projets qui se présentent.

16 En effet, dans la conduite d'un projet, l'inspiration, les relations domestiques, le renom, le civisme, le commercial et l'industriel doivent fonctionner ensemble, puisqu'une même personne doit conduire simultanément des actions impliquant créativité, cohérence étroite de l'équipe, soucis de l'intérêt général, attention permanente portée au client et efficacité productive. Ces différentes valeurs, ainsi que les compétences qui les accompagnent, ne sont toutefois pas entendues dans le sens qui caractérise les cités canoniques. Ainsi, dans le cadre d'un projet, la créativité est collective et non plus individuelle, comme dans le monde inspiré. De même, si la confiance et la personnalisation des relations se rapprochent de la cité domestique, la hiérarchie, le contrôle communautaire disparaissent ainsi que le caractère permanent des relations. Les relations commerciales jouent un rôle central, mais très différent de celui du monde marchand : les échanges sont intenses au cours du projet et pas ponctuels, largement interpersonnels et pas anonymes, les produits ou services ne sont pas préexistants mais coproduits dans le cadre de cette interaction. Enfin, si l'efficacité productive demeure un critère central, elle ne s'établit plus systématiquement sur les mêmes bases, les fonctions de chacun dans l'organisation étant beaucoup plus mouvantes et le contenu du travail ne pouvant être prescrit à l'avance faute d'être complètement défini. Le manager ou le chef de projet, capable de susciter l'adhésion de son équipe et de l'amener à se mobiliser autour du projet, remplace le traditionnel cadre, renvoyant surtout à une position statutaire dans une structure hiérarchie établie.

17 Selon L. Boltanski et E. Chiapello. les six mondes traditionnels ne seraient donc plus suffisants pour modéliser convenablement les motivations de l'action et ses justifications dans cette nouvelle l'orme organisationnelle, chacun perdant sa cohérence interne. C'est pour rendre compte de cette évolution qu'ils identifient une nouvelle cité, dite par projets donnant lieu à un monde organisationnel aux contours spécifiques. Le principe supérieur de ce monde, c'est-à-dire la valeur permettant de justifier et de motiver les actions est bien évidemment le projet, conçu comme activité dans un sens extrêmement large, dépassant le cadre professionnel délimité par un contrai de travail. La vie est perçue 
comme résultant d'une succession de projets, où les frontières entre le personnel et le professionnel, les loisirs et le travail, deviennent diffuses. Elle est fondée sur une multiplication de rencontres temporaires, liées aux projets et susceptibles de se renouveler au gré des circonstances. C'est l'activité qui est à l'origine de tout lien social, dans la mesure où c'est pour la mener à bien qu'est crée un collectif, pour une période déterminée. Les valeurs inhérentes à ce nouveau monde organisationnel s'expriment pour tous les intervenants potentiels en termes de capacité personnelles et non plus seulement professionnelles: autonomie, goût de la prise de risque, intuition, talent, capacité attirer la sympathie des autres, à inspirer confiance, goût du contact ${ }^{11}$ par exemple. Celte forme d'organisation, adaptée au capitalisme moderne, ne repose sur aucun des principes d'action traditionnels tout en empruntant à chacun d'eux, puisqu'elle vise à les intégrer dans un même ensemble.

Pour la justifier, il est nécessaire de faire référence à un paradigme plus général, afin de lui donner au moins une apparence de cohérence, pour l'expliquer, la rendre visible et lisible, et commencer à lui donner vie. Le paradigme du réseau est compatible avec les frontières mouvantes et la pluralité d'opérations temporaires et de projets qui caractérisent son activité. L'une des principales conditions à l'insertion dans le monde idéal-typique du projet consiste justement à être capable de prendre place dans des réseaux internes et externes aux organisations, conçus comme de véritables préalables pour participer à des projets et pour ne pas se faire exclure. La succession des projets permet en retour d'étendre le réseau en termes spatiaux, temporels et institutionnels, et assure à un individu adoptant le comportement adéquat de toujours trouver à s'insérer dans un projet.

Ce méta-monde articulé autour du projet ne nous semble toutefois pas faire preuve de la même cohérence que les autres mondes organisationnels précédemment définis, reposant sur des principes d'action établis de longue date. Le fait que la cité par projet n'ait été identifiée qu'à partir de l'analyse détaillée de textes de la littérature managériale des années 1990 sans mise en relation avec des œuvres de philosophie politique permettant d'en valider les principes nous semble totalement révélateur: le fonctionnement en réseau n'a pas véritablement été pensé ${ }^{12}$. Il est même possible de douter qu'une telle démarche soit possible. En effet, un réseau est capable de relier des éléments extrêmement diversifiés, en les plaçant sur le même plan et en mettant moins en avant leur nature que leurs relations. Les notions de singularité et de généralité, qui fondent les mondes organisationnels, sont fortement remises en question, de même que les grandeurs. Dans le fonctionnement réticulaire qui caractérise un projet, toutes les dimensions (créativité, commerce, efficacité...) sont d'égale importance et aucune ne prédomine. En définitive, ce monde du projet comporte une dimension tautologique: l'action est justifiée, expliquée, motivée par elle-même - le principe supérieur étant l'activité - et non pas en référence à des objectifs qui en sont l'aboutissement, comme l'innovation, l'enrichissement, l'intérêt général ou l'efficacité. On peut alors se demander si des projets, par définition tous différents même s'ils présentent des similitudes, sont véritablement susceptibles de donner lieu à un monde organisationnel aussi solide que les six autres, puisqu'ils ne peuvent véritablement constituer un idéal-type.

L'instabilité conceptuelle du monde organisationnel du projet reflète également une difficile mise en cohérence opérationnelle. En fait, la cité par projet et la référence réticulaire qui la fonde reflète surtout l'organisation telle qu'il faudrait qu'elle soit pour en assurer l'efficacité, et au-delà pour permettre la poursuite du processus d'accumulation 
dans le contexte économique actuel. Concrètement, comment faire pour obtenir une convergence des objectifs et des comportements des différents intervenants ? La présence du client joue évidemment un rôle central, la contrainte salariale également. Cependant, des dispositifs organisationnels et techniques permettent de lui donner forme: ils prennent en particulier forme dans les portails d'entreprises.

\section{Portails d'entreprises et intégration communicationnelle des mondes}

\section{Les portails et la mise en cohérence des rationalisations cognitives}

21 Il n'existe pas de définition du portail d'entreprise qui fasse l'unanimité. Il s'agit à la base d'un site intranet, le plus souvent placé en page d'accueil de-tous les navigateurs installés sur les postes de travail d'une société. Solutions informatiques paramétrables individuellement, les portails sont édités et commercialisés par les sociétés de services spécialisés en informatique, et notamment par les grands industriels comme SAP. PeopleSoft ou Microsoft qui éditent par ailleurs les ERP ${ }^{13}$. Un portail d'entreprise offre potentiellement accès à l'ensemble des ressources cognitives d'une organisation. Pour la société SAP. «Le portrait permet de simplifier, d'unifier, de personnaliser et de rendre plus flexible l'environnement de travail de chaque individu II valorise et rentabilise le capital aujourd'hui le plus précieux de l'entreprise la connaissance. (...)le portail définit chaque environnement en fonction du rôle de la personne II permet le développement de celle connaissance en facilitant l'échange et la collaboration, il donne enfin de la flexibilité au système, l'information permettant à ce dernier de devenir un outil d'aide au changement ${ }^{14}$ Les portails remplissent ainsi plusieurs fonctionnalités qui leur permettent d'intégrer tout à la fois les rationalisations cognitives et les mondes organisationnels: nous pouvons en présenter un rapide aperçu dans une perspective communicationnelle.

En premier lieu, les portails visent à favoriser les situations de communication dans le cadre du travail, afin de favoriser l'efficacité organisationnelle. Ils incorporent l'accès à la messagerie électronique, ainsi qu'à des systèmes de chat voire de travail collaboratif, permettant la participation simultanée de plusieurs personnes à la réalisation d'une même activité, quelle que soit leur localisation. Il s'agit ici de mettre en place un dispositif technique permettant de faire se rejoindre le monde inspiré, lié la production de connaissances nouvelles pour un projet, et le monde domestique, en cherchant à constituer un collectif pour renforcer les capacités créatives et favoriser la rencontre de domaines différents.

Les portails visent en second lieu à renforcer les processus de communication, en liaison avec les autres processus économiques centraux dans l'économie cognitive. Un portail offre ainsi accès à l'accès aux modules d'ERP depuis un poste de travail, et permet de démultiplier l'organisation en flux tendus par une automatisation des commandes, un meilleur suivi des clients, une adéquation plus directe entre l'offre et la demande et un accroissement de la réactivité. Les fonctionnalités de stockage et d'accès à l'information stockée dans l'entreprise - documents techniques ou professionnels, des cahiers de charges, des courriers échangés avec des partenaires économiques - s'inscrivent également dans ce cadre. L'enjeu est d'associer le monde industriel, marqué par l'efficacité de la production, même si les critères on changé par rapport à la période fordiste. et le monde marchand, qui n'est pas forcément directement compatible. L'automatisation du 
traitement de l'information prolonge l'automatisation de la production: les comportements professionnels sont quelque part subordonnés au système d'information, qui force le décloisonnement et la rencontre entre les mondes au nom de l'efficacité, par delà les identités professionnelles.

Enfin, les portails interviennent au niveau des politiques de communication et s'inscrivent de plein pied dans les démarches d'ingénierie symbolique qui assurent la mise en place de l'infrastructure idéologique permettant le fonctionnement de l'économie cognitive. Les solutions de gestion informatisée des ressources humaines peuvent cartographier les compétences et expériences des salariés. Au-delà des données en tant que telles, il s'agit de fournir des clés d'interprétation: en contactant un autre acteur, il est par exemple possible de comprendre les raisons et les modalités précises de choix d'une solution technique effectuée antérieurement, ou de son abandon lors d'un projet précédent. Le dispositif vient ainsi favoriser la constitution d'un réseau et offre les possibilités d'accéder à l'histoire partagée d'un collectif autour d'un projet, pleine d'éléments riches de sens mais demeurés tacites jusque là. Au-delà, les portails d'entreprise relaient la politique de communication interne, et tendent parfois à supplanter la presse d'entreprise. Le monde de l'opinion rejoint alors celui du renom, puisqu'il s'agit tout à la fois de diffuser l'image de l'entreprise et d'inciter fortement à la publication de documents de travail et aux débats sur des forums.

\section{Quelles perspectives?}

Les portails d'entreprises, souvent assimilés aux portails de knowledge management, relaient l'ensemble des dispositifs de rationalisation cognitive évoqués plus haut pour constituer une infrastructure favorisant le travail collectif, en visant à assurer une gestion communicutionnelle des connaissances, basée sur le développement d'environnements susceptibles défavoriser la mobilisation des connaissances au cours des activités de travail15Ils créent ainsi un monde organisationnel pour les projets, intégrant tant bien que mal les principes d'action traditionnels. Leur fonctionnement repose largement sur des démarches d'ingénierie symbolique conduisant à orienter les comportements et les représentations : au-delà du travail prescrit, on pourra parler du travail induit, par lequel la responsabilisation du salarié le conduit à réagir aux pressions croissantes de son environnement, conformément à ce qui est attendu de lui sous l'angle de la rationalité managériale ${ }^{16}$ Comme le déclarait Jeoren lient, directeur général de SAP France en 2000, le portail MySAP.com propose « un environnement organisé et apporte une force de normalisation du travail collaboratif». Il vise à "organiser les interactions entre les employés, à les faire collaborer et coopérer $»^{17}$ pour permettre "à des acteurs économiques de travailler sur une plate forme commune sans taire partie de la même société ${ }^{18}$.

Une organisation réticulaire ne peut comme on l'a vu fonctionner sans l'investissement volontaire des salaries et des acteurs qui la composent. Malgré la normalisation et la formalisation des procédures, tout ne peut pas être prescrit : il s'agit en l'ail d'impulser et d'encadrer une auto organisation qui aille dans le sens souhaité pour la satisfaction des objectifs organisationnels. Les objectifs de l'organisation doivent en l'ait être intériorisés, pour donner lieu à une véritable responsabilisation individuelle et à un investissement volontaire dans les objectifs généraux. Il s'agit de "faire accoucher une organisation: cristalliser une vision, élaborer un projet, créer une adhésion, entraîner, transformer et entretenir l'adhésion. Comme un homme politique, il va (...) falloir travailler votre circonscription, battre le 
trottoir, faire les marchés.(...). évangéliser. argumenter, discuter, rencontrer, téléphoner, séduire et menacer ». La communication est considérée comme un outil essentiel de ce changement organisationnel : "il s'agit de comprendre la logique de changement et les logiques, rarement cohérentes, des différents acteurs concernés pour concevoir et mettre en ceuvre une stratégie efficace de persuasion voire de propagande $»^{19}$.

Quels rôles la communication joue-t-elle pour la coexistence des nouveaux mondes organisationnels? Situations, processus et politiques de communication se trouvent au cœur des enjeux visant à l'intégration des différents mondes organisationnels. à assurer leurs tensions, pour assurer un consensus organisationnel minimal, En ce sens, le paradigme du réseau doit être examinée dans toutes ses implications : certes, un réseau est source d'autonomie, de liberté, de multiplicité des parcours possibles, pour l'homme connexionniste qui parcoure la cité par projets. Mais en même temps, un réseau est une structure qui encadre. Par sa structure, telle qu'elle peut être matérialisée dans un intranet, les parcours possibles sont guidés, l'autonomie placée sous contrôle. Dans un monde organisationnel fondé sur le réseau, il n'y a plus véritablement d'employeurs et de salariés, mais des individus qui sont en relation afin d'assurer une prestation et doivent se l'aire confiance. Tous sont en théorie placés sur un pied d'égalité. Conceptuellement. il n'y a pas de place pour des rapports sociaux, à fortiori asymétriques. En outre, ce réseau est au service d'un être extérieur : le client. Dès lors, il devient difficile de s'extraire d'un tel environnement et de le contester, à moins de nier la pression concurrentielle et la nécessité de demeurer compétitif et de ne plus être digne de confiance.

Une démarche communicationnelle vise à montrer comment les phénomènes de communication constituent une composante de l'action individuelle et collective, mais aussi comment ils dessinent les mondes d'action. Elle permet de tisser des liens entre actions en situation, technologies, enjeux, idéologies et d'étudier les influences respectives des différents modes de production du sens, des savoirs, des connaissances, d'interprétation de la réalité en relation avec des évolutions plus macroéconomiques et sociales.

\section{BIBLIOGRAPHIE}

BOLTANSKI. L.. CHIAPELLO. E.. Le nouvel esprit du capitalisme. Gallimard. NRE. 1999

BOLTANSKI. L.. THEVENOT. L.. De la justification. Les économies de la grandeur. Gallimard. NRF. 1991

BOUILLON., J.L., Du partage des savoirs à « l'économie cognitive » : quelles rationalisations informationnelles et communicationnelles ? in actes du colloque Partage îles savoirs. Université Lyon 3 - ERISCO. 28 février et $1^{\mathrm{er}}$ mars 2003

BOUILLON., J.L... Vers une approche communicationnelle de la gestion des connaissances, thèse pour le doctorat en SIC. Université de Toulouse le Mirail / LERASS. 1999.

BOUILLON..J.L... GARDERE. E.. « Définir les contours d'une approche en termes de communication organisationnelle : quelle application à l'étude des dispositifs de management des 
connaissances ? » in les recherches sur les communications organisationnelles en débat. CERCOR. Université Rennes 2. 29. 30 novembre et $\mathrm{I}^{\mathrm{er}}$ décembre 2001. p. 54-60

CHANTEAU..J.P.. La dimension socio-cognitive des institutions en économie : le rôle des représentations sociales dans la régulation. Actes $d u$

Forum de la Régulation 2001. Paris. CD ROM. 2001.

DE CRESCENZO. J.C.. Pratiques communicationnelles dans les réseaux informels de salariés. Actes préparatoires aux 4èmes Journées d'Etudes du Groupe d'Etudes et de Recherches sur les Communications Organisationnelles (SI SIC). Castres. 7-8-9 juin 2001. pp. 151-155.

DELCAMBRE. P.. (Ed). Communications organisationnelles objets, pratiques, dispositifs. Presses Universitaires de Rennes. 2000.

FLORIS. B., La gestion symbolique entre ingénierie et manipulation.

Sciences de la Société n 50-5l. 2000. pp. 173-195.

LAVOSY. O.. La communication au travail. Presses Universitaires de Grenoble, coll. La communication en plus. 2002.

LE MOENNE. C. (Ed). Communications d'entreprises et d'organisations. Presses universitaires de Rennes. 1998

MAYERE. A.. Mutations organisationnelles et évolutions des systèmes et activités d'information communication. IIDR en SIC. Université de Toulouse le Mirail / LERASS. 2001.

MUSSO P.. Critique des réseaux. PUF, coll. La politique éclatée. 2003 ORG\&CO. Communication organisante et organisation communicante : confrontations et dynamiques. Actes préparatoires aux 4èmes Journées d'Etudes du Groupe d'Etudes et de Recherches sur les Communications Organisationnelles (SESIC). Castres. 7-8-9 juin 2001

ORG\&CO. «Les recherches en communications organisationnelles en débats », actes préparatoires au colloque organisé par le Croupe d'Etudes et de Recherches sur les Communications Organisationnelles (SFSIC). Rennes. 20 novembre - $\mathrm{I}^{\mathrm{er}}$ décembre 2001

PETIT. P., les NTIC dans l'entreprise : une lente révolution des formes de concurrence. Actes du Forum de la Régulation 2001. Paris. CI) ROM. 2001. RAMAI IX. C. La critique est-elle soluble dans le capitalisme L'année de la régulation. $\mathrm{n}^{\circ}$ 5. 2001-2002

SALAIS. R.. (Ed.). Institutions et conventions : la réflexivité de l'action économique. Raisons Pratiques $n^{\circ}$. 1998.

\section{NOTES}

1. Boltanski. Thevenot. 1991 et Boltanski. Chiapello. 1999

2. Ramaux. 2002

3. Favereau. 1989. p.294. Référence explicite aux travaux de G. Bateson

4. Boltanski Chiapello p42 «Nous appelons esprit du capitalisme l'idéologie qui justifie l'engagement dans le capitalisme (...)Si le capitalisme non seulement a survécu contre les pronostics qui ont régulièrement annonce son effondrement mais n'a cesse d'étendre son empire, c'est bien aussi qu'il a pu prendre appui sur un certain nombre de représentations susceptibles de guider l'action - et de justifications partagées, qui le donnent pour un ordre acceptable et même souhaitable le seul possible, ou le meilleur des ordres possibles » p. 45

5. Notamment dans le cadre du groupe Org\&Co de la SFSIC 
6. Bouillon. Gardère. 2001

7. Note forum de la régulation

8. Bouillon. 2003

9. Bouillon. 1999

10. Une telle évolution a été promue par les gourous du Business Process Reengenering dans les années 1999

11. Boltanski. Chiapello. p.163 et sq

12. Musso. 2003

13. Nous avons engage il y a quelques mois un projet de recherche Au-delà de l'identification précise des composantes de ces offres, l'objectif est de repérer les conceptions de l'information, de la communication, des connaissances de l'organisation, qui sont sous-tendues. Cette démarche constitue aussi un repérage préalable a des investigations auprès des éditeurs eux-mêmes et dans des organisations en phase d'implantation de ces systèmes. L'accès au terrain implique en effet d'avoir saisi de manière Ires line les logiques en présences, les positions possibles des différents acteurs et de comprendre « leur » langage

14. http// www.sap.com/ France/solutions/portals/

15. Bouillon 1999

16. Olivesi 2002. Floris 2001

17. Les catégories d'outils collaboratifs. JDNet solutions. 28 octobre 2002 http.// solutions.joulnaldunet.com/0210/021028 Icolla shtml

18. Interview de Jeoren Bent. Directeur Général de SAP. JDNet solutions. 31 janvier 2000

19. "E-Approvisionnement et conduite du changement ", chronique d'Hubert d'Hondt. JDNet solutions. 11 octobre 2002.

http//solutions. journuldunet.com/0210/021011 chro dhondl shtml

\section{AUTEUR}

\section{JEAN-LUC BOUILLON}

Université Toulouse 3 - IUT 